удК 164

\title{
В.В. Целищев
}

\section{ИСТИННОСТЬ НЕРАЗРЕШИМЫХ ПРЕДЛОЖЕНИЙ В СВЕТЕ ПЕРВОЙ ТЕОРЕМЫ ГЁДЕЛЯ О НЕПОЛНОТЕ}

\author{
Исследование выполнено при финансовой поддержке РНФ (грант 16-18-10359).
}

\begin{abstract}
Сопоставляются два способа определения истинности неразрешимого геделева предложения. Стандартный способ апеллирует исключительно к метаматематическим соображениям, в то время как предложенный М. Даммитом анализ апеллирует к арифметической интерпретации. Экспликация аргумента Даммита и его опровержение ставят вопрос о различии эпистемологической значимости двух подходов к истинности геделева предложения.

Ключевые слова: неполнота; истина; геделево предложение; эпистемология; самореференция; Гёдель; Даммит.
\end{abstract}

Построение формальной системы для арифметики имеет целью «схватывание» интуитивных ее истин; это означает, что все истины должны быть доказуемы в этой системе. При достижении обычных в таком предприятии требований к системе, когда все доказуемые утверждения являются истинными, а все истинные - доказуемыми, мы имеем ситуацию, при которой истинные и доказуемые утверждения с эпистемологической точки зрения равноценны. В этом смысле полнота формальных систем является идеалом логического анализа оснований математики. Первая теорема Гёделя о неполноте арифметики утверждает, что для широкого класса формальных систем арифметики существуют неразрешимые предложения, являющиеся истинными. Таким образом, понятия истины в содержательной арифметике и формально доказуемого утверждения расходятся, что выливается в целый комплекс эпистемологических проблем. В фокусе этих проблем находится обоснование истинности так называемого геделевого предложения, истинного и недоказуемого в системе. Философский анализ этой проблемы имеет дело с пониманием роли способов конструирования геделева предложения, лежащих в основе конструирования идей и механизма кодирования и самоотносимости, балансирующих на грани парадокса. Прежде всего находящаяся на поверхности парадоксальность заключается в самой интерпретации геделевого предложения (далее - G).

Геделево предложение $\mathrm{G}$ в формальной системе $\mathrm{F}$, с интуитивной точки зрения, говорит: «Я недоказуемо в F». Доказуемо ли G в F? Пусть формальная система $\mathrm{F}$ обоснована, т.е. если предложение $\mathrm{G}$ выводимо в ней, тогда оно истинно в стандартной интерпретации. Но тогда видно, что $G$ невыводимо в F. Если бы оно было выводимо, тогда оно должно было бы быть ложным, так оно само говорит о своей невыводимости. Но в этом случае формальная система $\mathrm{F}$ не была бы обоснованной, что противоречит предположению. Далее, так как $\mathrm{G}$ недоказуемо в $\mathrm{F}$, и утверждает это обстоятельство, значит оно истинно [1. С. 61].

Такое «объяснение» истинности геделева предложения скрывает несколько важных обстоятельств. Некоторые исследователи полагают объяснение просто эвристическим замечанием самого Гёделя, требующим более тщательного рассмотрения, в частности устранения оттенка парадоксальности, связанным с идеей самореференции. Действительно, Гёдель впал в первородный грех, когда написал: «Мы, следовательно, имеем предложение, которое говорит о себе, что оно недоказуемо» [2. С. 148ff]. Это предложение может быть эвристическим, и, тем не менее, оно неверно. В работе нет самореференции и присутствует лишь простая или множественная диагонализация; эквивалентные неподвижные точки, но нет самореферентности в любом строгом смысле, который мы могли бы сделать точным. Мы не катаемся на тонком льду парадокса [3].

Булдту вторит П. Смит:

Нет ничего подозрительного или парадоксального в Первой теореме Гёделя, которая является техническим результатом о формальных аксиоматических системах (результат, который может быть доказан без обращения к «самоприменимым» предложениям) [4. С. 4].

Другим, лежащим на поверхности, уже реальным объяснением служит то обстоятельство, что в F можно доказать утверждение $\mathrm{G}$ истинно, если и только если $\mathrm{F}$ непротиворечива.

Отсюда следует, что истинность $G$ уже неявно предполагается в доказательстве в форме предположения о непротиворечивости F. Такое предположение подтверждается чисто математическими соображениями и методами, не оставляя места эпистемологическим соображениям. В какой мере вопрос об истинности гёделева предложения решается чисто математически? П. Смит дает достаточно развернутый ответ на этот вопрос, исследуя его на нескольких примерах формальных систем арифметики Q (арифметика Робинсона [5. С. 160-172]) и Арифметики Пеано (PA) [1. C. 166-169]. Система Q, основанная на первых четыpex постулатах Пеано (без аксиомы индукции), представляет прекрасный полигон для обсуждения результатов о неполноте, поскольку все четыре ее аксиомы рассматриваются как общепринятые арифметические истины и в ее основе лежит первопорядковая логика, она обладает всеми необходимыми ресурсами для конструирования геделева предложения. Другими словами, система $\mathrm{Q}$ вполне «респектабельна» и сконструированное в ней геделевское предложение $\mathrm{G}$ является истинным в силу этой респектабельности.

Другой случай - когда к системе Q мы добавляем в качестве дополнительной аксиомы какое-либо математическое утверждение или гипотезу, истинностное значение которого до сих пор неизвестно, скажем, догадку Гольдбаха. Результирующая система будет 
непротиворечивой, если догадка Гольдбаха истинна. Поскольку до сих пор этой догадке не нашлось контрпримера и есть другие соображения в пользу ее истинности, есть резоны считать построенное внутри новой системы геделево предложение «G (Гольдбах)» истинным.

Еще один случай - это система, образованная добавлением к аксиомам Арифметики Пеано (РА) аксиомы $\neg \mathrm{G}$ (отрицание $\mathrm{G}$ ). Эта теория, в силу интерпретации $\neg \mathrm{G}$ как ложного утверждения, не будет обоснованной, но при непротиворечивости РА она будет непротиворечивой, что позволяет конструировать геделево предложение $\mathrm{G}$, которое оказывается истинным по уже принятым ранее резонам. Из приведенных примеров Смит делает вывод, что у нас есть различные причины верить в обоснованность или непротиворечивость систем, и тем самым в истинность G. Это означает, что эта истинность определяется чисто математическими резонами [4. С. 171-173].

Следует отметить, что в последнем примере с добавлением в виде аксиомы отрицания геделева предложения мы получаем нестандартные интерпретации, что вряд ли может свидетельствовать в пользу самой идеи стандартной демонстрации истинности G. Кроме того, с нашей точки зрения, Смит делает поразительную ошибку, объясняя истинность $G$ разнообразием случаев непротиворечивости, которые приводят к многообразию геделевых предложений. Однако причина, по которой геделево предложение $\mathrm{G}$ является истинным, остается прежней, сводящейся к «эвристическому» замечанию Геделя. Фактически речь идет о том, что истинность $\mathrm{G}$ уже неявно предполагается в доказательстве в форме предположения о непротиворечивости соответствующей системы. П. Раатикайнен отмечает, что у нас есть столько же оснований полагать $\mathrm{G}$ истинным, сколько для веры в непротиворечивость формальной системы [6]. В известном смысле это релятивистский тезис, потому что вера в непротиворечивость варьируется от одной формальной системы к другой. Такой релятивизм вряд ли может быть удовлетворительным в отношении вопроса об истинности G. Он неоправдан как с математической точки зрения, так, с точки зрения эпистемологии.

Доказательство Гёделем своей Первой теоремы о неполноте осуществляется через конструирование того, что сейчас мы называем геделевым предложением. Примечательными особенностями геделевых предложений принято считать (i), что в некотором смысле они говорят сами о себе, что они недоказуемы; и (ii) что они истинны при условии непротиворечивости теории. Современные версии доказательства в стиле оригинального доказательства Гёделем - по крайней мере в стиле способа конструирования геделевого предложения - на самом деле не оказывают значительной поддержки этим тезисам или оказывают ее в лучшем случае ценой произвольных ограничений [7. C. 193].

Коль скоро непротиворечивость системы устанавливается синтаксически, аргумент в пользу истинности $G$ решается на синтаксическом уровне. Если же непротиворечивость устанавливается теоретикомодельным способом, тогда прежний способ демон- страции истинности геделевого предложения получает поддержку. Против обоих способов обсуждения выступает М. Даммит, который выдвинул интересные аргументы в пользу интуитивного происхождения истинности геделева предложения, имеющие важное эпистемологическое значение. Он указывает на фундаментальное обстоятельство, связанное с разным пониманием термина «модель».

Некоторые философы отрицают какое-либо содержание теоремы Гёделя, помимо синтаксического, полагая, что нет интуитивных причин называть неразрешимое предложение $\mathrm{G}$ «истинным»; но их мнение совершенно неправдоподобно. Ошибка состоит в неправильном применении понятия модели. Однако при обсуждении теоремы Гёделя мы оперируем понятием модели как нечто таким, которое может быть дано нам независимо от какого-либо описания: как вид интуитивной концепции, которую мы можем обозреть во всей ее полноте умственным взором... Это понятие не имеет ничего общего с концепцией, которая законно используется в математике [8. С. 190-191].

Геделево предложение по своей структуре является универсальной квантификацией. Как известно, при использовании неограниченной универсальной квантификации требуется осторожность, диктуемая финитистскими соображениями. Если такая квантификация понимается как пробегающая над полной бесконечной совокупностью чисел, тогда она должна интерпретироваться как гипотетическое утверждение: если задано некоторое число $\mathrm{n}$, тогда $\mathrm{P}(\mathrm{n})$ будет истинно для $\mathrm{P}(1), \mathrm{P}(2), \mathrm{P}(3)$ и т.д. При этом последовательность должна представлять потенциальную бесконечность утверждений. Это известное обстоятельство получает парадоксальное выражение в применении к геделеву предложению, структура которой представляет универсальную квантификацию. Г. Серени отмечает в этой связи следующее:

По определению, истинность геделева предложения влечет истинность его числовых конкретизаций. Так как эти конкретизации представляют собой $\Delta_{0}$ предложения, из теоремы о $\Sigma_{1}$-полноте следует, что все они доказуемы. Таким образом, доказуемость числовых конкретизаций геделева предложения следует из истинности самого предложения, которая, в свою очередь, следует из его недоказуемости. Следовательно, мы можем парадоксально заключить, в случае геделева предложения, что наше знание доказуемости всех его числовых конкретизаций следует из нашего знания недоказуемости предложения [9. C. 72-73].

Общепринятое объяснение истинности геделева предложения апеллирует именно к целостной конструкции универсальной квантификации. Между тем указанный парадокс и состоит в том, что для установления истинности геделевого предложения мы должны обратиться к нумерическим конкретизациям (числовым примерам) универсальной квантификации. Эта стратегия, предпринятая М. Даммитом, радикально меняет подход к установлению причин геделева предложения.

Этот вопрос связан с двумя важными идеями. Вопервых, это двойственный характер $\mathrm{G}$, которое может 
рассматриваться как с метаматематической, так и с арифметической точки зрения. Во-вторых, это идея, что истинность $\mathrm{G}$ устанавливается в контексте стандартной интерпретации формальной системы арифметики. Эти две идеи тесно связаны между собой.

Геделево предложение $G$ конструируется в формальной системе, которая призвана «схватить» концепцию натурального числа, и коль скоро G интерпретируется как истинное утверждение, в характеристике формализуемой части арифметики ничего не изменится существенно, если $\mathrm{G}$ добавить к аксиомам формальной системы в качестве аксиомы. Но вот если к этой системе добавить отрицание $\mathrm{G}$, тогда мы получаем формальную систему, которая характеризует не только натуральные числа, но и нечто выходящее за пределы ряда натуральных чисел. Речь идет о нестандартной интерпретации. Наличие нестандартной интерпретации является аргументом в пользу истинности G.

Р. Пенроуз отмечает в этой связи: «Заключение об истинности высказывания $\mathrm{G}(\mathrm{F})$ для непротиворечивой формальной системы F мы делаем, исходя из допущения, что те символы системы F, которые, как мы полагаем, служат для представления натуральных чисел, действительно представляют натуральные числа. Окажись на их месте другие числа, скажем, некие экзотические «сверхнатуральные» числа, - мы вполне могли бы обнаружить, что высказывание $\mathrm{G}(\mathrm{F})$ ложно» [10. С. 173].

Конечного способа убедиться в том, что «числа», о которых идет речь, являются натуральными, не существует. К этому факту прямое отношение имеют геделевские теоремы о неполноте. Любая аксиоматическая система, построенная для описания натуральных чисел, не позволяет нам определить, является ли $\mathrm{G}$ истинным или ложным. Но полагание системы непротиворечивой в этом отношении придает смысл истинности $\mathrm{G}$, который заключается в намеренной интерпретации символов системы. Таким образом, проблема определения истинностного значения геделева предложения напрямую связана с идеей стандартной интерпретации. Интерес представляет обращение этого тезиса, согласно которому истинность $\mathrm{G}$ и является признаком стандартной интерпретации. По такому пути и пошел в обосновании истинности G М. Даммит; этот подход ведет к значительной ревизии стандартных аргументов в пользу истинности геделевого предложения.

Поскольку G не доказуемо и не опровержимо, должны быть в системе такие модели, в которых оно истинно, и другие модели, в которых оно ложно. Поскольку $\mathrm{G}$ не истинно во всех моделях системы, мы можем распознать $\mathrm{G}$ как истинное предложение при условии, что под «истинным» имеется в виду «истинно в намеренной модели системы». Мы, таким образом, должны иметь в виду совершенно определенную идею того вида математической структуры, к которой мы намерены обращаться, когда говорим о натуральных числах; и через обращение именно к этой интуитивной концепции мы распознаем истинность G [8. C. 186].

Идея Даммита состоит в том, что истинность геделева предложения устанавливается обращением к ин- туитивно известным арифметическим истинам, которые представляют конкретизации универсальной квантификации. Поскольку предложение $\mathrm{G}$ имеет форму $(\forall \mathrm{x}) \mathrm{Ax}$, и коль скоро все $\mathrm{A}(0), \mathrm{A}(1), \mathrm{A}(2), \ldots$ признаются истинными, вполне обоснован переход от истинности нумерических конкретизаций к истинности $(\forall x) A x$. Однако этот переход совершается в рамках формализма, согласно первопорядковой логике, лежащей в основе формальной системы, и вряд ли он, в силу своей тривиальности, может претендовать на объяснение истинности $(\forall x) A x$. Скорее, на это может претендовать принцип, согласно которому все предложения $\mathrm{A}(0), \mathrm{A}(1), \mathrm{A}(2), \ldots$ истинны. Опять-таки, это предположение связано с различным пониманием модели, о котором говорилось выше, а именно модели в математическом смысле и модели как «реальности», благодаря которой они могут быть определены как истинные.

Обсуждение этого факта в терминах моделей затемняет этот в противном случае очевидный факт, потому что утверждением об истинности в некоторой модели всех предложений $\mathrm{A}(0), \mathrm{A}(1), \mathrm{A}(2), \ldots$ мы смазываем зазор между способностью распознавания для каждого $\mathrm{n}^{*}$ истинности $\mathrm{A}\left(\mathrm{n}^{*}\right)$ и способностью распознавания того факта, что для каждого $\mathrm{n}^{*} \mathrm{~A}(\mathrm{n} *)$ истинно [8. С. 192].

(Здесь $\mathrm{n}^{*}$ представляет цифру (numeral) числа n).

Условие непротиворечивости для истинности $\mathrm{G}$ устанавливается, по Даммиту, принципом, который апеллирует к тому, что выходит за рамки формальной системы. Принцип этот состоит в истинности всех $\mathrm{A}(0), \mathrm{A}(1), \mathrm{A}(2), \ldots$ Но именно это обстоятельство и означает непротиворечивость системы. Указанный принцип, высказанный Даммитом в несколько туманной манере, более четко сформулирован С. Клини:

«Если мы предполагаем, что теоретико-числовая формальная система должна быть непротиворечивой, мы можем распознать, что $\mathrm{G}$ истинно, приняв во внимание структуру этой системы как целого, хотя мы не можем распознать истинность $\mathrm{G}$ использованием только принципов вывода, формализованных в этой системе» [11. С. 426].

Собственно аргумент Даммита в пользу истинности $\mathrm{G}$ довольно краток, будучи представлен буквально одним абзацем: «Всякий раз, когда для некоторого предиката Вх мы можем распознать все утверждения В(0), $\mathrm{B}(1), \mathrm{B}(2), \ldots$ как истинные в стандартной модели, мы можем распознать $(\forall \mathrm{x}) \mathrm{Bx}$ как истинное в этой модели. Этот факт известен нам в силу нашей ясной интуитивной концепции структуры модели» [8. С. 191].

Необходимая экспликация идеи Даммита осуществлена Н. Теннантом [12] с помощью « семантического аргумента». Не входя в детали его аргументации, приведем его формулировку в следующем виде:

«G есть универсальная квантификация примитивно-рекурсивного предиката, где каждая нумерическая конкретизация предиката для любого числа доказуема в системе S. Доказательство в S гарантирует истинность. Отсюда, каждая конкретизация G истинна. Поэтому, поскольку $\mathrm{G}$ является просто универсальной квантификацией над подобными конкретизациями, геделево предложение должно быть истинным. 
Теннант при этом апеллирует к дефляционной теории истины, что лишь запутывает, или по крайней мере, затемняет аргументацию в пользу истинности G. Более успешную экспликацию аргумента Даммита (правда, с целью опровержения ее), следуя реконструкции аргумента К. Райтом, осуществил Г. Серени» [9. С. 50].

Нотация и основные факты, требуемые для понимания реконструкции аргументации Даммита, таковы. Как и в любом описании роли геделева предложения, имеем: для любой формулы $\varphi,{ }^{*} \varphi *$ обозначает геделев номер $\varphi$. Формальная система $\mathrm{S}$ есть произвольное, но фиксированное рекурсивное непротиворечивое расширение системы Q Робинсона, чья стандартная модель обозначается через $\omega$. Далее:

Proof $_{\mathrm{S}}=\left\{(\mathrm{m}, \mathrm{n}) \in \varphi^{2}: \mathrm{n}=* \varphi^{*}\right.$ для некоторой формулы $\varphi ; \mathrm{m}$ есть геделево число доказательства в $\mathrm{S}$ формулы $\varphi$.

Proof $_{\mathrm{s}}(\mathrm{x}, \mathrm{y})$ есть $\Delta_{0}$ формула, определяющая Proof s B $\omega$.

$\operatorname{Pr}_{\mathrm{S}}(\mathrm{y})=(\exists \mathrm{y}) \operatorname{Proof}_{\mathrm{S}}(\mathrm{x}, \mathrm{y})$

(Здесь и далее выражение * ${ }^{*}$ означает геделев номер выражения х).

Последняя формула является $\Sigma_{1}$-формулой, и поэтому для любой формулы $\operatorname{Pr}_{\mathrm{S}}(\varphi), \operatorname{Pr}_{\mathrm{S}}\left({ }^{*} \varphi^{*}\right)$ истинна точно в том случае, когда $\varphi$ доказуема в $\mathrm{S}$. Согласно Диагональной Лемме [13], имеем важное соотношение для геделева предложения $\mathrm{G}$ :

$\mathrm{Q}$ доказывает $\mathrm{G} \leftrightarrow \neg \operatorname{Pr}_{\mathrm{S}}\left({ }^{*} \mathrm{G}^{*}\right)$.

Поскольку система $\mathrm{S}$ обоснована по определению, $\mathrm{G}$ истинна точно в том случае, когда $\neg \operatorname{Pr}_{\mathrm{S}}\left({ }^{*} \mathrm{G}^{*}\right)$ и, следовательно, когда истинно $(\forall \mathrm{x}) \neg$ Proof $\mathrm{S}\left(\mathrm{x},{ }^{*} \mathrm{G}^{*}\right)$. Это означает важный факт: три выражения, а именно $\mathrm{G}, \neg \operatorname{Pr}_{\mathrm{S}}\left(* \mathrm{G}^{*}\right)$ и $(\forall \mathrm{x}) \neg \operatorname{Proof} \mathrm{S}\left(\mathrm{x},{ }^{*} \mathrm{G}^{*}\right)$, взаимозаменяемы. Однако статус этих утверждений разнится, поскольку одни являются метаматематическими, а другие - арифметическими. Это обстоятельство играет существенную роль при анализе аргументации Даммита. Действительно, при указанной взаимозаменяемости возникает вопрос, какой характер имеет геделево предложение $\mathrm{G}$ : метаматематический или же арифметический. Если имеет место второе, тогда истинность G доказывается через нумерические конкретизации G. Если же имеет место первый вариант, тогда $\mathrm{G}$ есть sui generic предложение, т.е. с эпистемологической точки зрения, имеющее особый характер. Г. Серени полагает, что аргументация Даммита не выдерживает критики при ее экспликации. Стратегия Серени состоит в сопоставлении двух экспликаций аргумента об истинности $\mathrm{G}$ - стандартной и Даммита. Цель Серени состоит в демонстрации того, что аргументация Даммита на самом деле, при кажущемся фундаментальном различии, сводится к стандартному варианту. Рассмотрим структуру обеих экспликаций.

Одна из экспликаций аргумента Даммита принадлежит К. Райту [14]. Райт дал свою версию для интуиционистской логики. Г. Серени модифицировал версию Райта для классической логики [9. С. 51]:

Для достаточно богатой формальной системы $\mathrm{S}$ (начиная с системы Q) имеем:

(1) $\operatorname{Proof}_{\mathrm{S}}\left(\mathrm{m},{ }^{*} \mathrm{G}^{*}\right)$ истинно $\rightarrow$ Посылка, $\mathrm{m}-$ натуральное число
(2) $\mathrm{S}$ доказывает Proof $\left(\mathrm{m},{ }^{*} \mathrm{G}^{*}\right) \rightarrow(1), \Sigma_{1}$-полнота $\mathrm{S}$

(3) $\mathrm{S}$ доказывает $\mathrm{G} \rightarrow(1)$, определение Proof $_{\mathrm{S}}$

(4) $\mathrm{S}$ доказывает $\neg \operatorname{Pr}_{\mathrm{S}}\left({ }^{*} \mathrm{G}^{*}\right) \rightarrow(3)$, определение $\mathrm{G}$

(5) $\mathrm{S}$ доказывает $\neg \operatorname{Proof}_{\mathrm{S}}\left(\mathrm{m},{ }^{*} \mathrm{G}^{*}\right) \rightarrow(4)$, определение $\operatorname{Pr}_{\mathrm{S}}$

(6) $\mathrm{S}$ доказывает $\operatorname{Proof}_{\mathrm{S}}\left(\mathrm{m},{ }^{*} \mathrm{G}^{*}\right) \& \neg \operatorname{Proof}_{\mathrm{S}}(\mathrm{m}$, $\left.* \mathrm{G}^{*}\right) \rightarrow(2),(5)$

(7) $\neg$ Proof $_{\mathrm{S}}\left(\mathrm{m},{ }^{*} \mathrm{G}^{*}\right)$ истинно $\rightarrow(6)$, противоречие c (1)

(8) $\neg \operatorname{Proof}_{\mathrm{S}}\left(\mathrm{n},{ }^{*} \mathrm{G}^{*}\right)$ для каждого $\mathrm{n} \in \omega \rightarrow(7)$, универсальное обобщение

(9) $(\forall \mathrm{x}) \neg \operatorname{Proof}_{\mathrm{S}}\left(\mathrm{x},{ }^{*} \mathrm{G}^{*}\right)$ истинно $\rightarrow(8)$, определение истинности в $\omega$. $\left.* \mathrm{G}^{*}\right)$

(10) $\mathrm{G}$ истинно $\rightarrow(9)$, экв. G и $(\forall \mathrm{x}) \neg$ Proof $_{\mathrm{S}}(\mathrm{x}$,

Серени находит в такой экспликации фундаментальный изъян. В решающем переходе от (8) к (9) вводится переменная на основании правила Универсального Обобщения логики первого порядка. Хотя Серени признает формальную обоснованность применения этого правила, тем не менее он полагает, что его применение требует использования неявной посылки об однородности элементов, подлежащих обобщению. Другими словами, эти элементы должны обладать общим свойством. Тогда обобщение не зависит от индивидуальных элементов и представляет собой некоторую схему формального вывода. Это означает, что природа элементов не имеет значения и в представленном выводе переменная $x$ не предполагает подстановки вместо нее именно чисел. Серени приходит к важному заключению о том, в данном выводе нет реального обращения к числам, т.е. к тем самым нумерическим конкретизациям универсальной квантификации, о которой говорил Даммит.

Суть проблемы состоит в том, что в выводе участвуют два выражения: $\operatorname{PrS}\left({ }^{*} \mathrm{G}^{*}\right)$ и $\operatorname{Proof}_{\mathrm{S}}\left(\mathrm{m},{ }^{*} \mathrm{G}^{*}\right)$ в качестве взаимозаменяемых выражений. Но если второе из них является арифметическим утверждением с участием геделева числа $\mathrm{m}$, то первое - метаматематическим утверждением. Использование эквивалентности их в выводе при переходе от (4) к (5) приводит к путанице. На самом деле, коль скоро в выводе нет явного обращения к числам, следует исключить $\operatorname{Proof}_{\mathrm{S}}\left(\mathrm{m},{ }^{*} \mathrm{G}^{*}\right)$ из структуры доказательства. В этом случае аргумент Даммита принимает следующий вид:

(1) $\operatorname{Pr}_{S}\left(* G^{*}\right)$ истинно $\rightarrow$ Посылка

(2) $\mathrm{S}$ доказывает $\operatorname{Pr}_{\mathrm{S}}\left({ }^{*} \mathrm{G}^{*}\right) \rightarrow(1), \Sigma_{1}$-полнота $\mathrm{S}$

(3) $\mathrm{S}$ доказывает $\mathrm{G} \rightarrow(1)$, определение $\operatorname{Pr}_{\mathrm{S}}$

(4) $\mathrm{S}$ доказывает $\neg \operatorname{Pr}_{\mathrm{S}}\left({ }^{*} \mathrm{G}^{*}\right) \rightarrow(3)$, определение $\mathrm{G}$

(5) $\mathrm{S}$ доказывает $\operatorname{Pr}_{\mathrm{S}}\left({ }^{*} \mathrm{G}^{*}\right) \& \neg \operatorname{Pr}_{\mathrm{S}}\left({ }^{*} \mathrm{G}^{*}\right) \rightarrow(2)$, (4)

(6) $\mathrm{S}$ доказывает $\neg \operatorname{Pr}_{\mathrm{S}}\left({ }^{*} \mathrm{G}^{*}\right)$ истинно $\rightarrow(1),(5)$ противоречит (1)

Однако этот вывод является экспликацией стандартного вывода недоказуемости геделева предложения и, в конечном счете, обоснованием его истинности. Это означает опровержение идеи Даммита об обосновании истинности $\mathrm{G}$ через нумерические конкретизации.

Каков при этом получается выигрыш в философской интерпретации Первой теоремы Гёделя? Аргумент Даммита о природе истинности геделева пред- 
ложения является альтернативным по отношению к стандартному объяснению этой истинности. Опровержение аргумента Даммита, предложенное Серени, основано на том, что экспликация этого аргумента раскрывает то обстоятельство, что он на самом деле сводится к стандартному. Не рассматривая аргументы в пользу стандартного объяснения, следует обратить внимание, какой ценой достигается такая редукция. Если цена оказывается слишком высокой, тогда и опровержение находится под вопросом.

Прежде всего, в чем состоит эпистемологическое различие двух подходов? Стандартный подход апеллирует к истинности геделева предложения как целого, в то время как подход Даммита апеллирует в этом вопросе к истинности нумерических конкретизаций предложения. Эпистемологическая дилемма в этом случае состоит в определении того, что первично: само $\mathrm{G}$ или его числовые примеры $\mathrm{G}(0), \mathrm{G}(1), \mathrm{G}(2), \ldots$ ? Cерени полагает, что объяснение универсальной квантификации $(\forall \mathrm{x}) \mathrm{Bx}$ через примеры, что и является обоснованием логического правила Универсального Обобщения, в принципе неверно. Он полагает, что в реальной математике универсальная квантификация не имеет места, поскольку в ней оперируют числами; более того, $(\forall \mathrm{x})$ является логическим артефактом. Ситуация становится запутанной именно в связи с геделевым предложением. Как только, используя идею геделевской нумерации, стало возможным кодировать арифметические утверждения в виде самих арифметических утверждений, т.е. в виде метаматематических утверждений, роль $(\forall \mathrm{x})$ становится более сложной.

Действительно, для обычного арифметического предложения в формальной теории никаких сложностей нет, но для геделева предложения они есть.

Двойственная природа универсального арифметического предложения проявляет себя в том факте, что суждения, выражаемые двумя различными формулировками утверждения его истинности, которые эпистемологически неразличимы в случае обычного арифметического предложения, становятся эпистемологически различными суждениями. Действительно, если $\mathrm{M}=(\forall \mathrm{x}) \varphi \mathrm{x}$ такое предложение, тогда утверждение, что М истинно может быть интерпретировано способом, который радикально отличен от обычной арифметической интерпретации, согласно которому функция $\varphi(\mathrm{n})$ истинна для каждого натурального числа. В самом деле, можно верить (или знать), что М истинно, без знания (или даже не подозревая), что М есть универсальное утверждение и что у него вообще есть арифметическая интерпретация. Например, можно верить, что Арифметика Пеано свободна от противоречий; следовательно, опираясь на общее знание сообщества логиков, что предложение Con (PA) выражает непротиворечивость РА, можно верить, что Con(PA) истинно, без осознания того факта, что это на самом деле предложение формальной арифметики [9. С. 64-65].

Разные эпистемологические стандарты для обычных арифметических утверждений и для метаарифметических утверждений объясняются тем, что геделево предложение включает в себя два утверждения, сформулированных на двух разных языках: языке арифметики и языке метаарифметики. В первом случае мы имеем обыденную арифметику, а во втором кодированные утверждения. Осознание двойственного характера геделева предложения позволяет заключить, что его истинность может быть доказана двумя путями: как чисто арифметического утверждения и как метаматематического утверждения. Если с формальной точки зрения они равноценны, за счет эквивалентности $\operatorname{Pr}_{\mathrm{S}}\left({ }^{*} \mathrm{G}^{*}\right)$ и $\operatorname{Proof}_{\mathrm{S}}\left(\mathrm{m},{ }^{*} \mathrm{G}^{*}\right)$, то с эпистемологической точки зрения вопрос состоит в том, имеет ли доказательство метаматематическое приоритет над доказательством арифметическим, которое получается рассмотрением числовых примеров.

С одной стороны, арифметический аргумент является эпистемологически предпочтительным, потому что апелляция к числовым примерам является интуитивно понятной. С другой стороны, можно полагать, что метаматематический аргумент (или стандартный аргумент) более приемлем опять-таки эпистемологически, потому что арифметический предикат, входящий в геделево предложение, столь сложен, что вряд ли можно говорить о его интуитивном содержании и тем более его постижении человеком.

Предпринятые экспликации аргумента Даммита в пользу истинности геделева предложения не могут считаться заключительными, поскольку оставляют открытыми значительное число вопросов. Соотношение метатеоретических и арифметических методов включает вопросы самореферентных утверждений, различных способов доказательства Первой теоремы Гёделя, роль понятия непротиворечивости в связи с истинностью геделева предложения, способы конструирования геделева предложения, роль кодирования, и т.д. Это подлинный клубок проблем, которые являются в настоящее время предметом интенсивных исследований феномена неполноты.

\section{ЛИТЕРАТУРА}

1. George A., Velleman D. Philosophies of Mathematics. Oxford : Blackwell Publishing, 2002.

2. Godel K. On Formally Undecidable Propositions of Principia Mathematica and Related Systems I// Collected Works of Kurt Godel. Vol. 1 / eds. Feferman S. et al. Oxford : Oxford University Press, 1986. P. 144-195.

3. Buldt B. Godel's Incompleteness Theorems - Tutorial. URL: http://opus.ipfw.edu/philos_facpres/130

4. Smith P. An Introduction to Godel's Theorem (second edition). Cambridge : Cambridge University Press, 2013.

5. Boolos G., Jeffrey R. Computability and Logic. Cambridge : Cambridge University Press, 1989.

6. Raatikainen P. On the Philosophical Relevance of Godel’s Incompleteness Theorems // Revue Internationale de Philosophie. 2005. Vol. 59, № 4. P. 513-534.

7. Milne P. On Godel Sentences and What They Say // Philosophia Mathematica (III). 2007. Vol. 15. P. $193-226$.

8. Dummett M. The Philosophical Significance of Godel's Theorem // Truth and Other Enigmas. Cambridge : Harvard University Press, 1978. P. 186-201. 
9. Sereny G. How Do We Know that the Godelian Sentence of a Consistent Theory is True? // Philosophia Mathematica (III). 2011. Vol. 19. P. 4773.

10. Пенроуз Р. Тени разума. М. : Ин-т компьют. исслед., 2003.

11. Клини С.К. Введение в метаматематику. М. : Изд-во иностр. лит., 1957.

12. Tennant N. Deflationism and the Godel Phenomena // Mind. 2002. Vol. 111, № 443. P. 551-582.

13. Hajek P., Pudlak P. Metamathematics of First-Order Arithmetic. Berlin : Springer, 1993.

14. Wright C. About 'The Philosophical Significance of the Godel's Theorem'; Some Issues // McGinnes B., Oliveri (eds.) The Philosophy of Michael Dummett. Dordrecht : Kluwer AcademicPress, 1994. P. 167-202.

Статья представлена научной редакцией «Философия» 5 мая 2017 г.

\section{THE TRUTH OF UNDECIDABLE SENTENCES IN THE PERSPECTIVE OF GODEL'S FIRST INCOMPLETENESS} THEOREM

Vestnik Tomskogo gosudarstvennogo universiteta - Tomsk State University Journal, 2017, 421, 53-58.

DOI: $10.17223 / 15617793 / 421 / 7$

Vitaly V. Tselishchev, Institute of Philosophy and Law, Siberian Branch of Russian Academy of Sciences (Novosibirsk, Russian Federation). E-mail: leitval@gmail.com

Keywords: incompleteness; truth; Godelian sentence; epistemology; self-reference; Godel; Dummett.

The first theorem of Godel on the incompleteness of arithmetic asserts that for a wide class of formal systems of arithmetic there are true but unsolvable sentences. Thus, the concepts of truth in informal arithmetic and formally provable statements diverge, which gives rise to the whole complex of epistemological problems. The purpose of the article is to analyze the problems of the truth of the unprovable Godel sentence, in particular, to consider the role of methods for constructing the Godel sentence, the ideas and the mechanism of coding and self-reference, balancing on the verge of a paradox. The standard establishment of the truth of the Godel sentence in a syntactic and model-theoretic way was challenged by M. Dummett, who advanced interesting arguments in favor of the intuitive origin of the truth of the Godel sentence, which has an important epistemological significance. The author proposes a comparison of the standard approach with Dummett's understanding of the nature of the Godel proposition. The problem solved in the article: the use of the dual nature of the Godel sentence in the context of establishing its truth as (a) the metamathematical sentence and (b) the arithmetic assertion; proof of the truth of the Godel proposition as a universal quantification by establishing the truth of its examples; demonstration of the need for a standard interpretation of the formal system of arithmetic for the truth of the Godel sentence; establishing a connection between these two ideas. Methods used: reconstructing Dummett's arguments using first-order logic; use of the metamathematical result on the relation of the Godel proposition of the provability predicate in the Diagonal Lemma, or the Fixed Point Theorem. Conclusions: Dummett's argument about the nature of the truth of the Godel sentence is alternative with respect to the standard explanation of this truth. The argument of G. Sereni on the reducibility of Dummet's argument to the standard way of establishing the truth of the Godel sentence is investigated. The reason for reducibility is the existence of different epistemological standards for the usual arithmetic and meta-arithmetic assertions, since the sentence can be interpreted in a dual way - as arithmetic and as metamathematical. The comparison of Dummett's and Sereni's arguments rests on the assumption of the incomprehensibility of the arithmetic interpretation of the predicative of provability and the sufficiency of the metamathematical interpretation of the Godel sentence. It is shown that the undertaken explication of Dummett's argument in favor of the truth of the Godel sentence can not be considered as a final, since it leaves a considerable number of questions open. The relation between metatheoretical and arithmetic methods includes the questions of self-referential assertions, various ways of proving the First Theorem of Godel, the role of the concept of consistency in connection with the truth of the Godel sentence, the ways of constructing the Godel sentence, the role of coding, etc. This is a genuine tangle of problems that are currently the subject of intense research into the phenomenon of incompleteness.

\section{REFERENCES}

1. George, A. \& Velleman, D. (2002) Philosophies of Mathematics. Oxford: Blackwell Publishing.

2. Godel, K. (1986) On Formally Undecidable Propositions of Principia Mathematica and Related Systems I. In: Feferman, S. et al. (eds) Collected Works of Kurt Godel. Oxford: Oxford University Press.

3. Buldt, B. (2015) Godel's Incompleteness Theorems - Tutorial. [Online] Available from: http://opus.ipfw.edu/philos_facpres/130.

4. Smith, P. (2013) An Introduction to Godel's Theorem. 2nd ed. Cambridge: Cambridge University Press.

5. Boolos, G. \& Jeffrey, R. (1989) Computability and Logic. Cambridge: Cambridge University Press.

6. Raatikainen, P. (2005) On the Philosophical Relevance of Godel's Incompleteness Theorems. Revue Internationale de Philosophie. 59:4. pp. 513534.

7. Milne, P. (2007) On Godel Sentences and What They Say. Philosophia Mathematica (III). 15. pp. $193-226$.

8. Dummett, M. (1978) Truth and Other Enigmas. Cambridge: Harvard University Press. pp. 186-201.

9. Sereny, G. (2011) How Do We Know that the Godelian Sentence of a Consistent Theory is True? Philosophia Mathematica (III). 19. pp. 47-73.

10. Penrose, R. (2003) Teni razuma [Shadows of Reason]. Translated from English. Moscow: Institute of Computer Studies.

11. Kleene, S.C. (1957) Vvedenie v metamatematiku [Introduction to metamathematics]. Moscow: Izd-vo inostr. lit.

12. Tennant, N. (2002) Deflationism and the Godel Phenomena. Mind. 111:443. pp. 551-582.

13. Hajek, P. \& Pudlak, P. (1993) Metamathematics of First-Order Arithmetic. Berlin: Springer.

14. Wright, C. (1994) About 'The Philosophical Significance of the Godel's Theorem'; Some Issues. In: McGinnes, B.F. \& Oliveri, G. (eds.) The Philosophy of Michael Dummett. Dordrecht: Kluwer AcademicPress. 